\section{Cardiac defects and altered ryanodine receptor function in mice lacking FKBP12}

Weinian Shou ${ }^{\star}$, Bahman Aghdasi $\dagger$, Dawna L. Armstrong ${ }^{\star}$, Qiuxia Guo*, Shideng Bao*, Min-Ji Charng Lawrence M. Mathews $₫$, Michael D. Schneider $\neq$, Susan L. Hamilton $\dagger$ \& Martin M. Matzuk ${ }^{\star} \|$

Departments of ${ }^{\star}$ Pathology, $\|$ Cell Biology, 9 Molecular and Human Genetics, $\dagger$ Molecular Physiology and Biophysics, and $\$$ Medicine, Baylor College of Medicine, Houston, Texas 77030, USA

$\S$ Department of Biological Chemistry, University of Michigan, Ann Arbor, Michigan 48109, USA

FKBP12, a cis-trans prolyl isomerase that binds the immunosuppressants FK506 and rapamycin, is ubiquitously expressed and interacts with proteins in several intracellular signal transduction systems ${ }^{1}$. Although FKBP12 interacts with the cytoplasmic domains of type I receptors of the transforming growth factor- $\beta$ (TGF- $\beta$ ) superfamily in vitro, the function of FKBP12 in TGF- $\beta$ superfamily signalling is controversial ${ }^{2-6}$. FKBP12 also physically interacts stoichiometrically with multiple intracellular calcium release channels including the tetrameric skeletal muscle ryanodine receptor $(\mathrm{RyR} 1)^{7,8}$. In contrast, the cardiac ryanodine receptor, RyR2, appears to bind selectively the FKBP12 homologue, FKBP12.6 (refs 9, 10). To define the functions of FKBP12 in vivo, we generated mutant mice deficient in FKBP12 using embryonic stem (ES) cell technology. FKBP12-deficient mice have normal skeletal muscle but have severe dilated cardiomyopathy and ventricular septal defects that mimic a human congenital heart disorder, noncompaction of left ventricular myocardium ${ }^{11,12}$. About $9 \%$ of the mutants exhibit exencephaly secondary to a defect in neural tube closure. Physiological studies demonstrate that FKBP12 is dispensable for TGF- $\beta$-mediated signalling, but modulates the calcium release activity of both skeletal and cardiac ryanodine receptors.

FKBP12 exons 3 and 4 encode functional domains involved in FK506 and rapamycin binding ${ }^{13}$, cis-trans prolyl isomerase activity $^{13}$, and TGF- $\beta$ family type 1 receptor binding ${ }^{2,3}$. To generate a null mutation, a targeted deletion $\left(f k b p 12^{\mathrm{ml}}\right)$ of these key exons was generated using ES cell technology (Fig. 1a). Heterozygous $\left(f k b p 12^{\mathrm{ml}} /+\right)$ mice were viable and fertile and were intercrossed to obtain FKBP12-deficient ( $\left.f k b p 12^{\mathrm{ml}} / f k b p 12^{\mathrm{ml}}\right)$ mice (Fig. 1b). Genotype analysis of $376 \mathrm{~F}_{2} \mathrm{C} 57 \mathrm{BL} / 6 \mathrm{~J} / 129 \mathrm{SvEv}$ hybrid and $181 \mathrm{~F}_{2}$ $129 \mathrm{SvEv}$ inbred offspring at weaning demonstrated only 8 viable FKBP12-deficient mice. The distribution of genotypes at embryonic day 14.5 (E14.5) (88 wild-type (25\%), 177 heterozygotes (51\%), and 84 homozygotes $(24 \%))$ and at E18.5 (220 wild-type (30\%), 390 heterozygote $(53 \%)$, and 125 homozygotes $(17 \%))$ suggested that the majority of the mutant mice died between E14.5 and birth.

To determine the causes of this embryonic and neonatal lethality, embryos derived by caesarean section were examined morphologically and histologically. The majority of FKBP12-deficient mutants died between E14.5 and birth because of severe dilated cardiomyopathy and ventricular septal defects (VSD). At E18.5, the majority of the FKBP12-deficient mice gasped for breath, but continued to exhibit pallor despite normal haematocrit, and demonstrated a rapid demise suggesting a failure of perfusion. Some of these E18.5 FKBP12-deficient mice were oedematous, and the majority had dramatically enlarged hearts because of four-chamber dilation (Fig. 2a, b). The FKBP12-deficient heart weight $(13.4 \pm 0.44 \mathrm{mg})$ was greatly increased compared to controls $(8.14 \pm 0.38 \mathrm{mg})$ $(P<0.0005)$. Detailed histological analysis revealed multiple abnormal anatomical structures in FKBP12-deficient hearts (Fig. $2 \mathrm{c}, \mathrm{d}$ ). which resembled the human congenital heart disorder, noncompaction of left ventricular myocardium, which is often accompanied by ventricular septal defects ${ }^{11,12}$. In E14.5 control hearts (Fig. 2c), the ventricular walls were of roughly equal thickness and trabeculated. However, the FKBP12-deficient hearts at E14.5 and E18.5 exhibited prominent ventricular septal defects, increased cavity diameters, thinner left ventricular walls, hypertrophic trabeculae, and deep intertrabecular recesses (Fig. $2 \mathrm{~d}$ and data not shown), indicating that the condensation of myocardium is disrupted in the FKBP12-deficient hearts. This myocardial noncompaction appears to be independent of the ventricular septal defect because other mouse mutants with ventricular septal defects do not have myocardial noncompaction ${ }^{14,15}$. Consistent with these defects, in situ hybridization revealed that FKBP12 is expressed at high levels throughout the embryonic heart including endocardium, myocardium, and septum (Fig. 2e). Lastly, consistent with this cardiac dysfunction, severe haemorrhage and necrosis were observed in the livers of many FKBP12-deficient mutants (Fig. 2f, $\mathrm{g}$ ), mimicking the liver centrolobular necrosis seen in passive congestive heart failure patients.

In addition to the cardiac defects, $9 \%(8 / 84)$ of the E14.5 and $4 \%$ (5/125) of the E18.5 FKBP12-deficient embryos had exencephaly

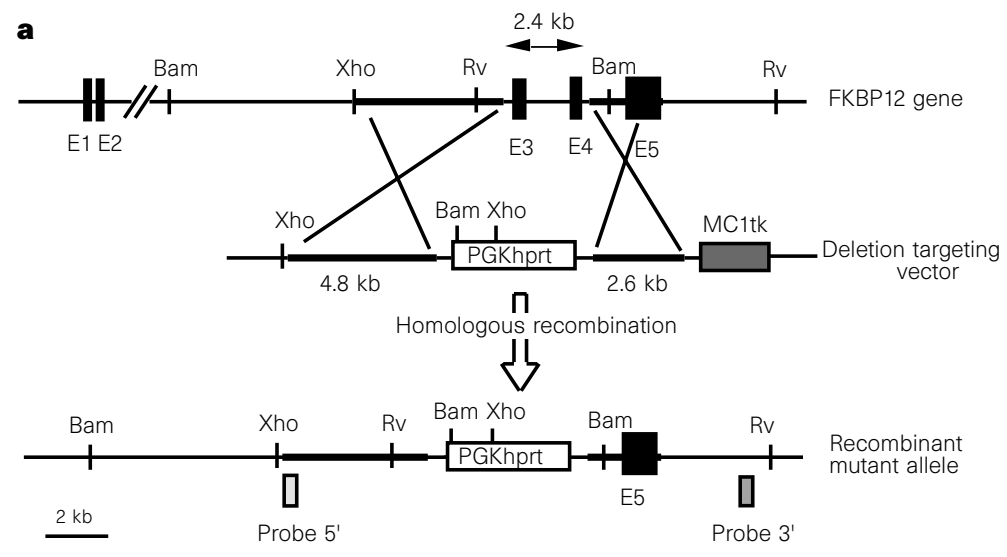

Figure 1 Generation of FKBP12-deficient mice. Targeting vector to mutate the mouse FKBP12 gene in ES cells (a) and Southern blot analysis of DNA derived from a single litter of E18.5 embryos from matings of $f k b p 12^{\mathrm{ml}} /+$ mice $(\mathbf{b})$. Genomic DNA $(\sim 5 \mu \mathrm{g})$ was digested with Bam $\mathrm{H} 1$ and hybridized with a $5^{\prime}$ probe.
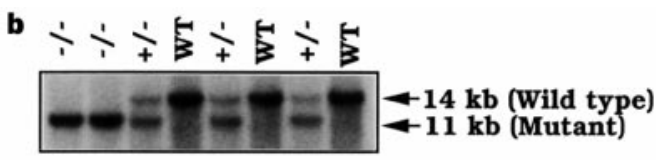

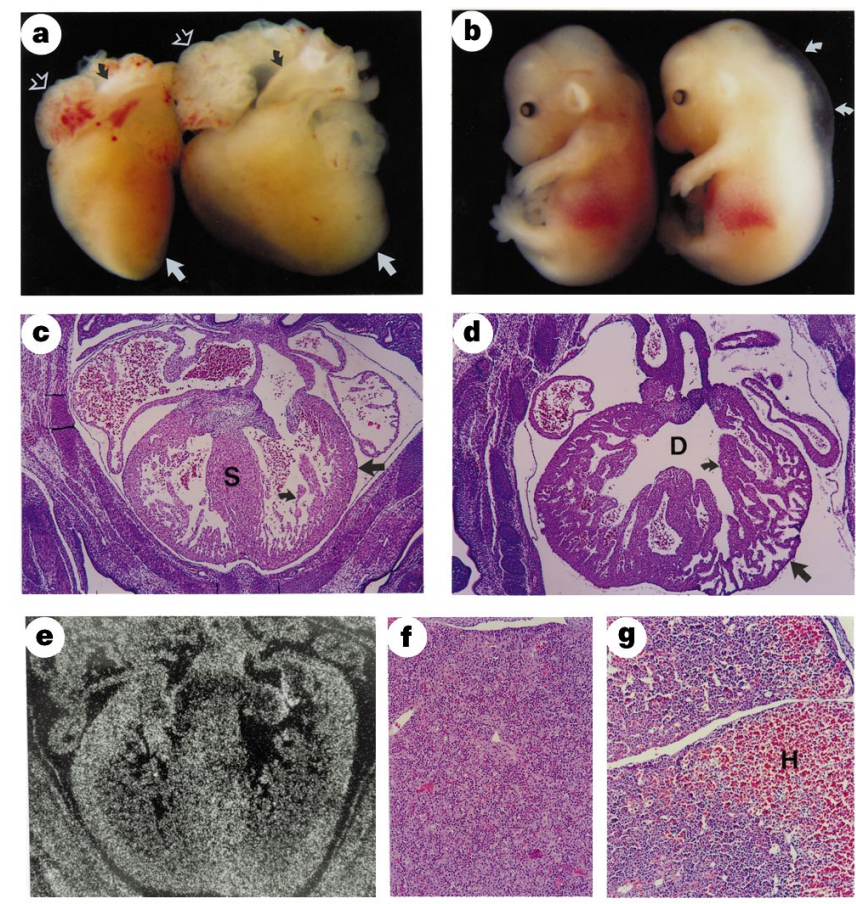

Figure 2 Cardiac and liver analysis of wild-type and FKBP12-deficient mutants. a, The E18.5 FKBP12 mutant heart (right) is enlarged compared to the wild-type heart (left). Atria (right atrium, open arrows), ventricles (left ventricle, solid white arrows) and outflow track (small black arrow) are roughly doubled in size in the mutant. b, About 50\% of E14.5 mutant embryos are oedematous (right, arrows) consistent with an early heart defect ${ }^{14,15}$. c, d, Histological analysis of E14.5 FKBP12-deficient (d) and littermate control hearts (c). The two ventricular walls (large arrow, left ventricle) of the control heart (c) are of roughly equal thickness and slightly trabeculated (small arrow). The ventricular septum (S) is prominent. In the FKBP12-deficient heart (d), there is a thinned left ventricular wall (large arrow), a prominent ventricular septal defect (D) and hypertrophic trabeculae (small arrow) associated with deep intertrabecular recesses. e, In situ analysis of FKBP12 mRNA expression in the mouse E13.5 heart. f, g. Wild-type control (f) and FKBP12-deficient $(\mathbf{g})$ E14.5 livers. Haemorrhage $(H)$ and necrosis are present in the mutant liver.
(Fig. 3a, b). Cranial neural tube closure is complete by E9.0 in wildtype embryos, but in $9 \%(6 / 65)$ of the FKBP12-deficient E9.5 embryos, neural tube closure defects were present (Fig. 3c, d). Histologically, these mutants demonstrated normal elevation and apposition of the neural folds as well as normal mesenchyme underlying the folds, which is distinct from twist- and cart-1deficient mice in which disrupted mesenchyme leads to neural tube defects ${ }^{16,17}$.

As mentioned above, eight FKBP12-deficient mice survived to weaning, and seven of these died within a few weeks because of an apparent cardiac-related wasting syndrome (the eighth lived to 14 months; see below). Both male and female FKBP-12-deficient mice had normal sexual differentiation indicating intact Müllerianinhibiting substance (MIS) and activin signalling pathways ${ }^{18}$. These mutants did not have anaemia or pancytopenia or any lymphocyte defects based on complete blood cell analysis and
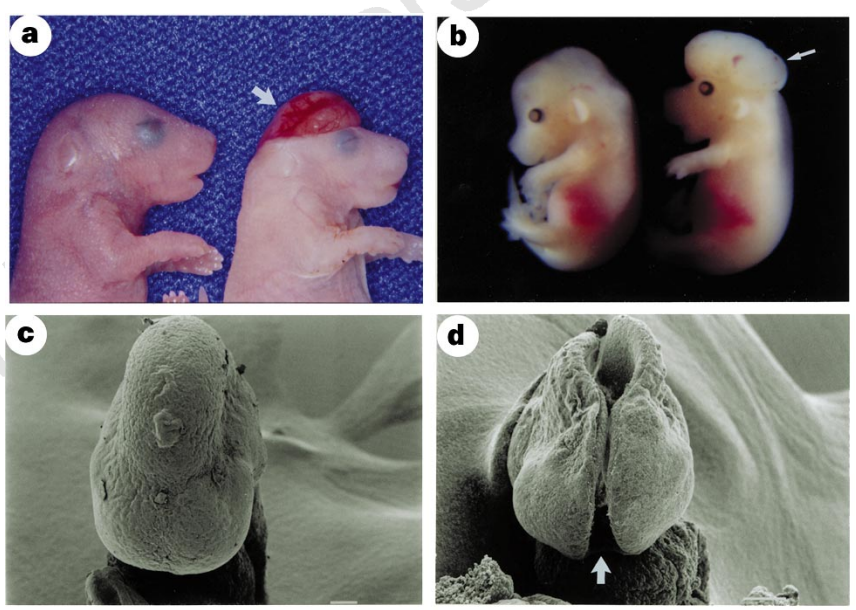

Figure 3 Analysis of wild-type and FKBP12 exencephaly mutants. a, 4\% of E18.5 FKBP12 mutants have exencephaly (right) in contrast to control littermates (left). b, Lateral view of E14.5 wild-type (left) and FKBP12-deficient (right) embryos. Note the 'cauliflower-like' protrusion of the mutant brain (arrow). c, d, Scanning electron microscopy of wild-type (c) and FKBP12-deficient (d) E9.5 embryos. The cranial neural tube is completely closed in the wild-type (c) but remains open in the mutant (d, arrow).

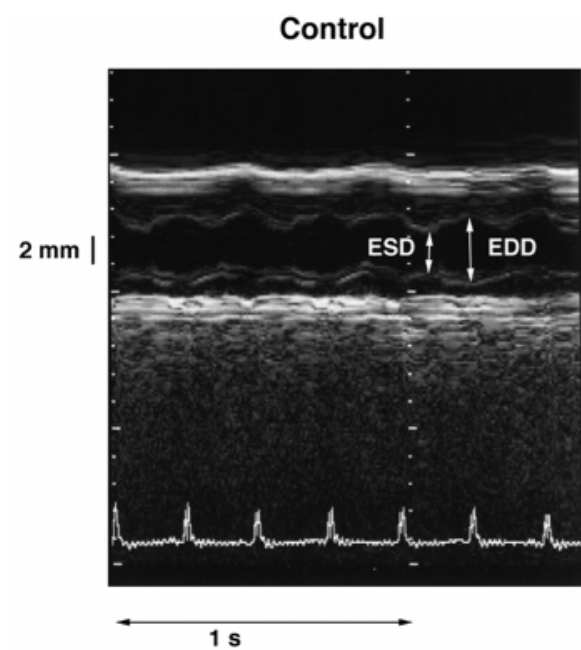

Figure 4 Cardiac performance of adult mice. Transthoracic $\mathrm{M}$ mode echocardiographic tracings ${ }^{19}$ in a control (left) and an FKBP12-deficient (right) mouse. Vertical arrows indicate left ventricular (LV) chamber at end-diastolic diameter (EDD) and

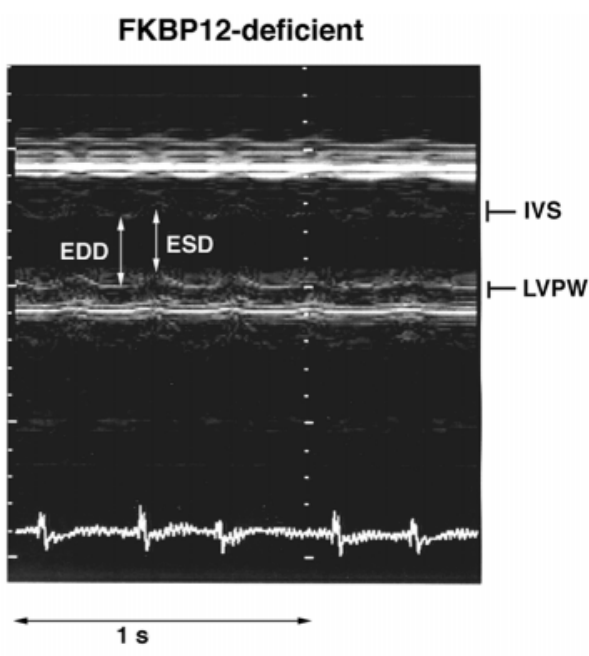

end-systolic diameter (ESD). Heart rate is indicated at the bottom of each panel. IVS, Intraventricular septum; LVPW, left ventricular posterior wall. 
fluorescent-activated cell sorting (FACS) analysis, and the only abnormality was the presence of melanin-containing cells in the spleens of six out of eight mutant mice. Echocardiographic analysis $^{19}$ of a 14-month-old FKBP12-deficient male mouse revealed a ventricular septal defect (data not shown) and a dilated left ventricular chamber (that is, end-diastolic diameter (EDD) was greater in the FKBP12-deficient mouse $(5.93 \pm 0.10 \mathrm{~mm})$ versus the age-matched control $(4.93 \pm 0.08 \mathrm{~mm})$ mouse $(n=5$ heart beats; Fig. 4)). The mutant heart showed diminished fractional shortening $(\% \mathrm{FS}=19.9 \%)$ and ejection fraction $(\% \mathrm{EF}=35.8 \%)$ compared to the control heart $(\% \mathrm{FS}=41.6 \% ; \% \mathrm{EF}=65.1 \%)$ indicating a depression of contractile activity in the ventricular wall of the mutant heart, similar to MLP-deficient mice ${ }^{20}$. Thus, the cardiac phenotype of this FKBP12-deficient mouse mimics the clinical features of cardiomyopathy and heart failure in humans.

FKBP1 2 was postulated to inhibit activin and TGF- $\beta$ signalling by binding to type I TGF- $\beta$ /activin receptors ${ }^{2,3,7}$ suggesting that TGF$\beta$-mediated signalling should be enhanced in FKBP12-deficient cells. However, transfection assays with a TGF- $\beta$-inducible p3TPLux plasmid showed that the induction of luciferase by TGF- $\beta$ is not significantly different between FKBP12-deficient and control cells (Fig. 5). Thus, the above-mentioned normal sexual differentiation in FKBP12-deficient male and female mice, and these in vitro transfection studies demonstrate that the in vitro interaction between FKBP12 and activin/TGF- $\beta$ /MIS type I receptors is not physiologically important. In addition, FKBP12-deficient mice do not phenocopy any of the reported TGF- $\beta$ superfamily ligand or receptor knockout or overexpressor mouse models ${ }^{18}$.

FKBP12 binds and modulates skeletal muscle RyR1 ${ }^{7,8}$ and was believed to play a role in skeletal muscle excitation-contraction coupling $^{21}$. However, in contrast to the excitation-contraction uncoupling and muscular degeneration phenotype of RyR1-deficient mice ${ }^{22}$, FKBP12-deficient mutants failed to demonstrate any gross, histological, or electron microscopic abnormalities in their skeletal muscle (data not shown). To analyse the functional properties of RyR1 function in the absence of FKBP12, skeletal muscle membranes from wild-type and FKBP12-deficient mice were prepared and reconstituted into planar lipid bilayers. RyR1 from FKBP12-deficient mice demonstrated an increased probability of opening compared to RyR1 from wild-type mice, and the channel exists mostly in subconductance states (not fully open) (Fig. 6a, b). RyR1 from wild-type mice remained in a closed state $84 \pm 2 \%$ $(n=3)$ of the time whereas RyR1 from FKBP12-deficient mice

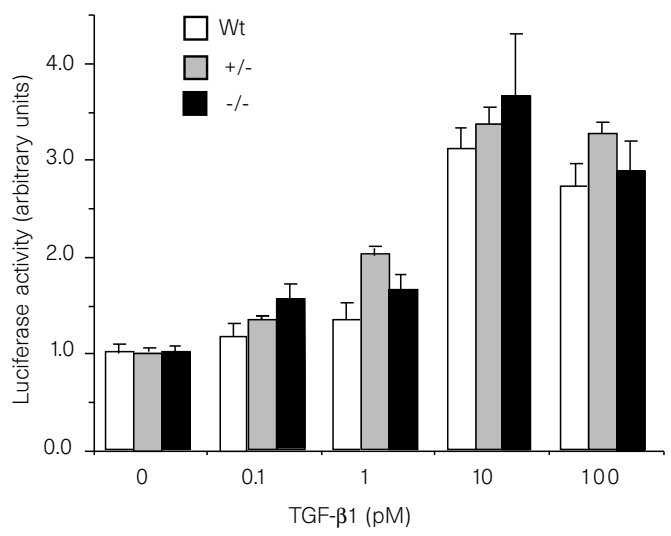

Figure 5 FKBP12 is not essential for TGF- $\beta$-mediated signalling. E14.5 fibroblasts isolated from FKBP12-deficient $(-/-)$, heterozygous $(+/-)$ and wild-type $(\mathrm{Wt})$ embryos were transfected with p3TP-Lux and induced by TGF- $\beta$ at concentrations indicated in the panel as described previously ${ }^{4}$. The relative luciferase activity in the absence of TGF- $\beta$ was set to one for the wild-type fibroblasts. There was no significant differences in the induction of luciferase (mean \pm s.e., $n=6)$ in the absence of FKBP12. remained closed only $16 \pm 4 \%(n=5)$ of the time. In addition, the probability of the channel existing at a subconductance state increased over five-fold in the mutants compared to the wild-type mice $(79 \pm 2 \%$ versus $13.8 \pm 4 \%$, respectively).

The FKBP12 orthologue, FKBP12.6, was believed to bind cardiac RyR2 selectively ${ }^{9,10}$. However, northern blot analysis of RNA from E18.5 skeletal and cardiac muscle demonstrated low levels of FKBP12.6 messenger RNA which were not upregulated in the mutants (data not shown). To determine whether the cardiac phenotype in FKBP12-deficient mice could be due to defects in cardiac RyR2 function, the single-channel behaviour of RyR2 was examined (Fig. 6c, d). Similar to the RyR1 findings, RyR2 from wild-type mice was closed $80 \pm 7 \%(n=3)$ of the time whereas RyR2 from FKBP12-deficient mice was closed $13 \pm 10 \%(n=4)$ of the time. Similarly, the amount of time RyR2 was found at a subconductance state increased over five-fold in the FKBP12deficient mice $(80 \pm 10 \%)$ versus control mice $(14.4 \pm 7 \%)$.

Most observations of FKBP12 functions have been based on experiments using an excess of immunosuppressive drugs (such as FK506 and rapamycin) and some of these conclusions have been contradictory. Our lipid bilayer experiments demonstrate that

\section{a RyR1, wild type}
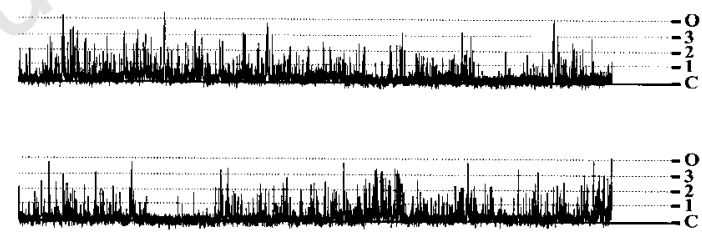

\section{b RyR1, FKBP12-deficient}
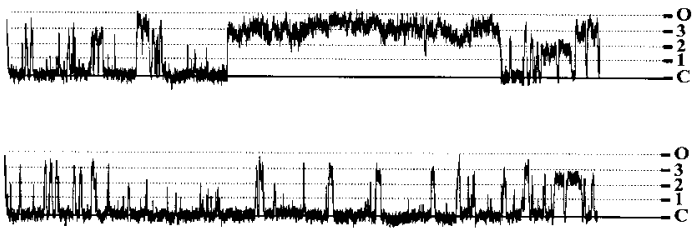

c RyR2, wild type
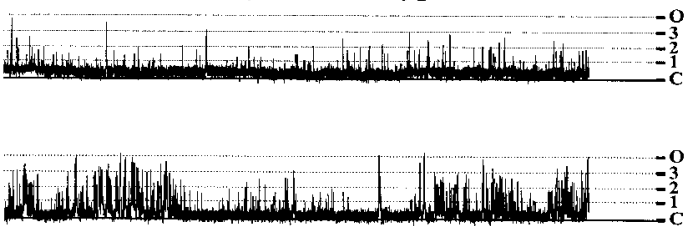

d RyR2, FKBP12-deficient
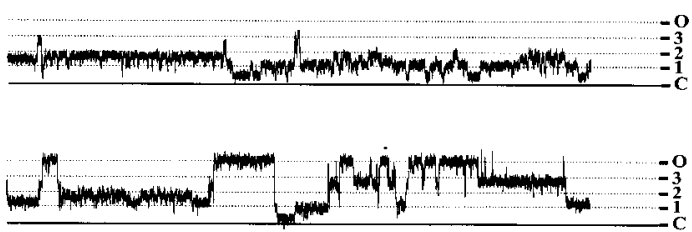

$10 \mathrm{pAL}$

$250 \mathrm{~ms}$ 
absence of FKBP12 alters the single-channel properties of both cardiac RyR2 and skeletal RyR1, that FKBP12.6 cannot functionally replace FKBP12, and that these defects may contribute to the cardiac phenotype in FKBP12-deficient mice. Increased frequency of substate channel openings suggests a model whereby FKBP12 is actively involved in the cooperative interactions between subunits of the ryanodine receptor tetramer as presented by Marks and colleagues ${ }^{8}$. Interestingly, the cardiac phenotype of FKBP12-deficient mutants correlates with the cardiac phenotype of humans and chickens treated with the immunosuppressant FK506. It has been reported ${ }^{23}$ that five paediatric organ transplant patients, who received high doses of FK506, developed hypertrophic cardiomyopathy associated with clinically severe heart failure that was reversed by switching immunosuppressant therapy from FK506 to cyclosporin. Likewise, it has been reported ${ }^{24}$ that FK506, but not cyclosporin A, induced cardiac enlargement in chick embryos which subsequently caused embryonic lethality. One enigma from our studies was that the absence of FKBP12 results in abnormal gating properties of both RyR1 and RyR2, yet only cardiac dysfunction is noted. This may reflect differences in excitation-contraction coupling mechanisms between cardiac and skeletal muscle. Cardiac calcium release channels are activated by calcium influx, whereas the skeletal channels are regulated in part by the transverse tubule voltage sensor $^{25}$. This extra level of control in skeletal muscle might act to prevent opening of the channel in the absence of excitation, thereby decreasing calcium leak from the sarcoplasmic reticulum. Changes in resting cytosolic calcium in cardiac tissue would contribute to the systolic and diastolic dysfunction of FKBP12-deficient embryonic and adult hearts, and subsequently lead to dilated cardiomyopathy and noncompaction of left ventricular myocardium. These FKBP12-deficient mice are an important model for the testing of pharmacological agents and other therapies that may retard or reverse human heart failure.

\section{Methods}

Targeted deletion. FKBP12 genomic clones and four FKBP12 pseudogenes were isolated from a mouse $129 \mathrm{SvEv}$ genomic library (Stratagene) using a human FKBP12 complementary DNA. To determine the chromosomal localization of the single-copy mouse FKBP12 gene, we used the Jackson Laboratory backcross DNA panel map service ${ }^{26}$. The mouse FKBP12 gene was localized to chromosome 2 , about $84 \mathrm{cM}$ from the centromere, and cosegregated with a group of 11 loci including D2Mit22 and Snta1, the syntenic region of human chromosome 20 where the human FKBP12 gene maps ${ }^{27}$. Linearized targeting vector $(25 \mu \mathrm{g})$ was electroporated into the hprt-negative AB2.1 ES cell line, cell clones were selected in HAT (hypoxanthine, aminopterine and thymidine) and FIAU (1-( $2^{\prime}$-deoxy- $2^{\prime}$-fluoro- $\beta$-D-arabinofuranosyl $)-5^{\prime}$ iodouracil), DNA from the clones was analysed by Southern blot, and targeted ES cell clone FK35-A12 was expanded and injected into blastocysts as described $^{28}$. Male chimaeras were bred to C57BL/6J or 129SvEv females to generate $\mathrm{F}_{1}$ offspring. To confirm that the $f k b p 12^{\mathrm{m} 1}$ mutation was null, northern and western blot analyses were performed. No FKBP12 mRNA or protein were observed in homozygous mutants $\left(f k b p 12^{\mathrm{ml}} / f k b p 12^{\mathrm{ml}}\right)$ when compared to wild-type controls (data not shown).

Histological, morphological and in situ hybridization analysis. Embryos and tissues were fixed in 10\% neutral buffered formalin, paraffin embedded and sectioned $(5 \mu \mathrm{m})$, and stained with haematoxylin and eosin. For scanning electron microscopy, E9.5 embryos were fixed in Karnovsky's fixative ( $\mathrm{pH} 7.5)$, rinsed in $0.1 \mathrm{M}$ phosphate buffer ( $\mathrm{pH} 7.3$ ), post-fixed in $1 \%$ phosphate buffered osmium tetroxide, dehydrated, and dried to critical point with carbon dioxide. The embryos were then coated with gold-palladium, and examined with a Jeol 6100 scanning electron microscope. In situ hybridization was performed as described in ref. 29. Mouse FKBP12 (coding sequence) antisense and sense probes were labelled with $\left[\alpha-{ }^{35} \mathrm{~S}\right] \mathrm{UTP}$ using a Promega Riboprobe System. The sense probe did not demonstrate any significant background signal.

Bilayer analysis for single channel activity. Sarcoplasmic reticulum membrane preparations were generated from E18.5 or adult mouse skeletal or cardiac muscle. Experiments were performed as previously described ${ }^{30}$ and repeated at least 3 times. Steady-state open probabilities $\left(P_{\mathrm{O}}\right)$ were determined by measuring the amount of time that the channel remained open during three minutes of recording. Using $\left[{ }^{3} \mathrm{H}\right]$ ryanodine, the binding site densities for wild-type skeletal, FKBP12-deficient skeletal, wild-type cardiac, and FKBP12-deficient cardiac tissue were $0.09 \pm 0.04(n=3), 0.10 \pm 0.02$ $(n=3), 0.15 \pm 0.05(n=3)$ and $0.20 \pm 0.04(n=3) \mathrm{pmol} \mathrm{mg}^{-1}$, respectively. Isolation of mouse embryonic fibroblasts and transfection assays. Primary fibroblasts were isolated from E14.5 mouse embryos, maintained in DMEM containing $10 \%$ FCS, plated at $1 \times 10^{5}$ cells per well in 12 -well plates, cultured overnight, transfected with p3TP-Lux and CMV- $\beta$-galactosidase reporter plasmids, treated with porcine TGF- $\beta 1$ (R\&D Systems), and analysed for luciferase and $\beta$-galactosidase activity $24 \mathrm{~h}$ after TGF- $\beta 1$ treatment as described $^{4}$. Western blot analysis of the wild-type fibroblasts demonstrated significant levels of FKBP12 protein (data not shown).

Received 8 August; accepted 28 October 1997.

1. Snyder, S. H. \& Sabatini, D. M. Immunophilins and the nervous system. Nature Med. 1, 32-37 (1995).

2. Wang, T. et al. The immunophilin FKBP12 functions as a common inhibitor of the TGF $\beta$ family type I receptors. Cell 86, 435-444 (1996).

3. Chen, Y.-G., Liu, F. \& Massagué, J. Mechanisms of TGF $\beta$ receptor inhibition by FKBP12. EMBO 13, 3866-3876 (1997).

4. Charng, M.-J., Kinnunen, P., Hawker,J., Brand, T. \& Schneider, M. D. FKBP12 recognition is dispensable for signal generation by type I transforming growth factor- $\beta$ receptors. J. Biol. Chem. 271, 22941-22944 (1996).

5. Okadome, T. et al. Characterization of the interaction of FKBP12 with the transforming growth factor- $\beta$ type 1 receptor in vivo. J. Biol. Chem. 271, 21687-21690 (1996).

6. Zimmerman, C. M. \& Mathews, L. S. Activin receptors: cellular signalling by receptor serine kinases. Biochem. Soc. Symp. 62, 25-38 (1996).

7. Jayaraman, T. et al. FK506-binding protein associated with the calcium release channel (ryanodine receptor). J. Biol. Chem. 267, 9474-9477 (1992).

8. Brillantes, A. B. et al. Stabilization of calcium release channel (ryanodine receptor) function by FK506binding protein. Cell 77, 513-523 (1994).

9. Timerman, A. P. et al. Selective binding of FKBP12.6 by the cardiac ryanodine receptor. J. Biol. Chem. 271, 20385-20391 (1996).

10. Lam, E. et al. A novel FK506 binding protein can mediate the immunosuppressive effects of FK506 and is associated with the cardiac ryanodine receptor. J. Biol. Chem. 270, 26511-26522 (1995).

11. Chin, T. K., Perlof, J. K., Williams, R. G., Jue, K. \& Mohrmann, T. Isolated noncompaction of left ventricular myocardium, a study of eight cases. Circulation 82, 507-513 (1990).

12. Ritter, M. et al. Isolated noncompaction of the myocardium in adults. Mayo Clin. Proc. 72, 26-31 (1997).

13. Van Duyne, G. D., Standaert, R. F., Karplus, P. A. \& Schrieber, S. L. Atomic structure of FKBP-FK506, an immunophilin-immunosuppressant complex. Science 252, 839-842 (1991).

14. Rossant, J. Mouse mutants and cardiac development, new molecular insights into cardiogenesis. Circ. Res. 78, 349-353 (1996).

15. Olson, E. N. \& Srivastava, D. Molecular pathways controlling heart development. Science 272, 671676 (1996).

16. Chen, Z.-F. \& Behringer, R. R. Twist is required in head mesenchyme for cranical neural tube morphogenesis. Genes Dev. 9, 686-699 (1995).

17. Zhao, Q., Behringer, R. R. \& Crombrugghe, B. Prenatal folic acid treatment suppresses acrania and meroanencephaly in mice mutant for the Cartl homeobox gene. Nature Genet. 13, 275-283 (1996).

18. Lau, A. L., Shou, W., Guo, Q. \& Matzuk, M. M. in Inhibin, Activin and Follistatin (eds Aono, T., Sugino, H. \& Vale, W. W.) 220-243 (Springer, New York, 1997).

19. Tanaka, N. et al. Transthoracic echocardiography in models of cardiac disease in the mouse. Circulation 94, 1109-1117 (1996).

20. Arber, A. et al. MLP-deficient mice exhibit a disruption of cardiac cytoarchitectural organization, dilated cardiomyopathy, and heart failure. Cell 88, 393-403 (1997).

21. Lamb, G. D. \& Stephenson, D. G. Effects of FK506 and rapamycin on excitation-contraction coupling in skeletal muscle fibers in the rat. J. Physiol. 494, 569-576 (1996).

22. Takeshima, H. et al. Excitation-contraction uncoupling and muscular degeneration in mice lacking functional skeletal muscle ryanodine-receptor gene. Nature 369, 556-559 (1994).

23. Atkison, P. et al. Hypertrophic cardiomyopathy associated with tacrolimus in paediatric transplant patients. Lancet 345, 894-896 (1995).

24. Okata, K. et al. The role of an immunophilin, FKBP12, in chick embryonic cardiac development. Circulation 94 (suppl. 1) 1-120 (1996).

25. Näbauar, M., Callewaert, G., Cleemann, L. \& Morad, M. Regulation of calcium release is gated by calcium current, not gating charge, in cardiac myocytes. Science 244, 800-803 (1989).

26. Rowe, L. B. et al. Maps from two interspecific backcross DNA panels available as a community genetic mapping resource. Mammal. Genome 5, 253-274 (1994).

27. Dilella, A. G., Hawkins, A., Craig, R. J., Schreiber, S. L. \& Griffin, C. A. Chromosomal band assignments of the genes encoding human FKBP12 and FKBP12.6. Biochem. Biophys. Res. Commun. 189, 819-823 (1992)

28. Bradley, A. Teratocarcinomas and Embryonic Stem Cells: a Practical Approach (ed. Robinson, E. J.) 113-151 (IRL, Oxford, 1987).

29. Albrecht, U., Eichele, G., Helms, J. A. \& Lu, H.-C. in Molecular and Cellular Methods in Developmental Toxicology (ed. Daston, G. P.) 23-48 (CRC, Boca Raton, 1997)

30. Aghdasi, B., Zhang, J.-Z., Wu, Y., Reid, M. \& Hamilton, S. L. Multiple classes of sulfhydryls modulate the skeletal muscle $\mathrm{Ca}^{2+}$ release channel. J. Biol. Chem. 272, 3739-3748 (1997).

Acknowledgements. We thank D. Kearney and M. Finegold for their expert opinions on the cardiac and liver pathology, J. Towbin for advice on congenital heart disease, K. Kurrelmeyer, T. Pham and G. Taffet for aid in echocardiographic analysis, J. Barrish for aid in electron microscopic analysis, S. Schreiber for the anti-FKBP12 antibody, J. Massague for the p3TP-lux plasmid, J.-Z. Zhang for aid in sarcoplasmic reticulum membrane preparation, S. Li for statistical help, T. R. Kumar and J. Towbin for their critical reading of the manuscript and S. Baker for aid in manuscript preparation. These studies were supported in part by National Institutes of Health grants (to M.M.M., S.L.H., M.D.S. and L.S.M.) and a Muscular Dystrophy Association grant (to S.L.H.)

Correspondence and requests for materials should be addressed to M.M.M. (email: mmatzuk@bcm. tmc.edu). 